\title{
Modélisation du transport dissous et particulaire dans l'estuaire de Morlaix. Application au devenir des bactéries entériques
}

\author{
P. Le Hir $\left({ }^{*}\right)$, J.F. Guillaud $\left({ }^{*}\right)$, M. Pommepuy $\left({ }^{*}\right)$, F. Le Guyader $\left({ }^{* *}\right)$, J.C. Salomon $\left({ }^{*}\right)$
}

\section{Introduction}

Les sites estuariens, souvent propices au développement de la conchyliculture, sont aussi soumis à de nombreux apports anthropiques qui peuvent altérer leur qualité sanitaire.

Les efforts économiques et techniques qui doivent être entrepris pour réduire la contamination bactériologique de ces zones nécessitent le développement d'outils capables de prédire le transport dissous et particulaire des bactéries fécales et d'intégrer le comportement (temps de mortalité, adsorption...) de ces mêmes bactéries en mer.

Une étude de ce type a été développée sur l'estuaire de Morlaix, localisé sur la côte nord de la Bretagne.

Afin de cerner le devenir des bactéries rejetées par une station d'épuration en amont de l'estuaire, un ensemble de modèles hydro-sédimentaires permettant de simuler le transport d'éléments dissous, des sédiments fins et des contaminants adsorbés a été développé. Ces modèles ont été intégrés pour constituer la base d'un logiciel de modélisation de la qualité de l'eau.
Parallèlement, des travaux bactériologiques ont été réalisés pour estimer :

- la contamination de la zone dans différentes conditions hydrodynamiques ;

- les temps de mortalité dans l'eau et dans les sédiments ; - le flux de bactéries fécales rejetées par les sources principales (rivière et station d'épuration) en distinguant les formes libres et liées, dont les comportements sont respectivement comparables à ceux d'éléments dissous ou particulaires.

Ces flux, ainsi que les temps de mortalité des bactéries, ont été introduits dans le modèle d'advection/dispersion pour réaliser des calculs de concentrations bactériennes que l'on a comparées aux concentrations mesurées dans l'estuaire.

(*) IFREMER. Centre de Brest, B.P. 70, 29280 Plouzané.

(**) UER des Sciences Médicales et Pharmaceutiques, avenue du Professeur L.-Bernard, 35043 Rennes Cedex.

\section{Modelling dissolved and particulate contaminants transport in the Morlaix estuary. Application to enteric bacteria discharge.}

Dissolved contaminants and cohesive sediment transport models have been developped and applied to a macrotidal estuary. These models are original as several dissolved and particulate constituents can be simultaneously computed, the later being followed in the surficial sediment according to the deposition events. Besides laboratory and field experiments have been conducted in order to evaluate the survival of enteric bacteria both within the sediment and the water body. Showing off rather long survival times especially in the sediment, the results have been introduced in the models in order to simulate the impact of urban disposal on water quality in a shellfishing area. 


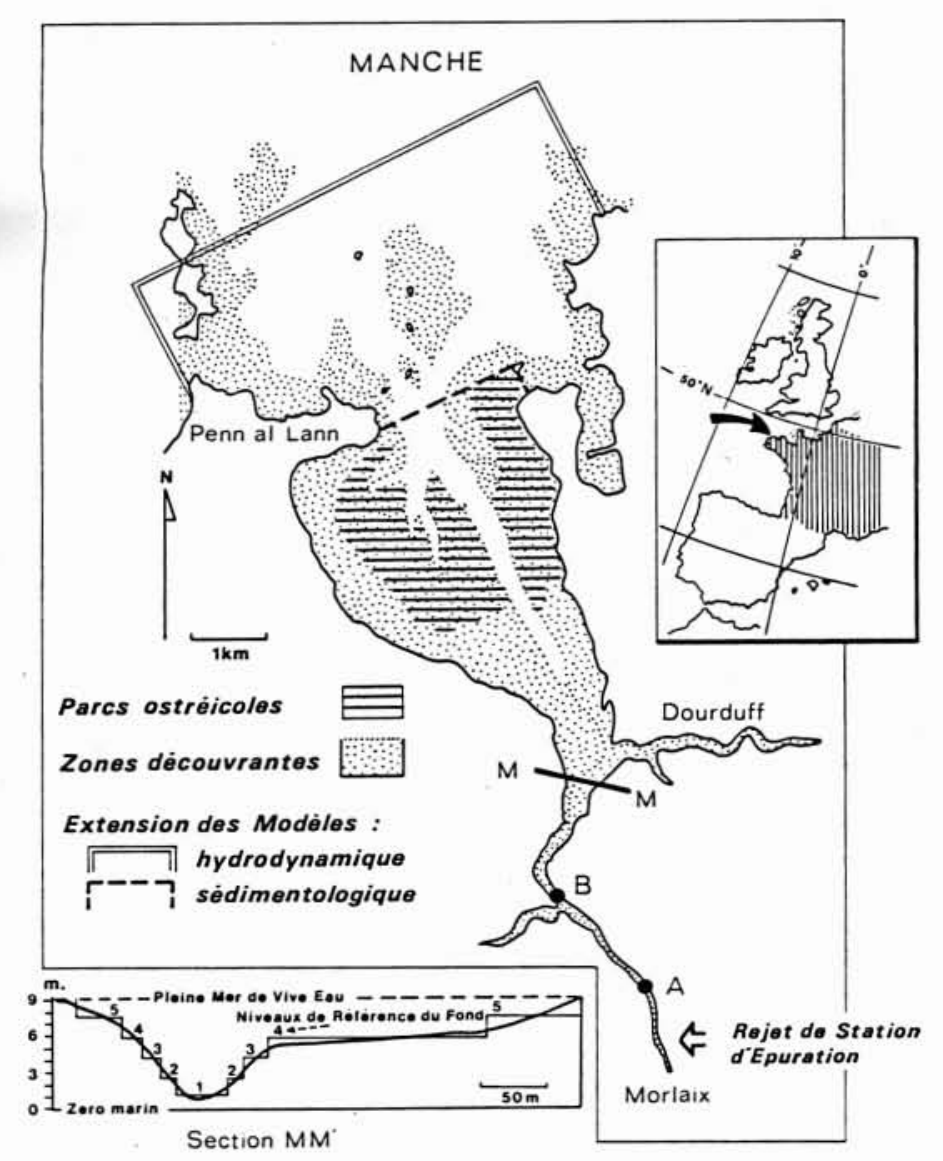

1. Présentation du secteur d'étude: l'estuaire et la baie de Morlaix.

\section{Présentation de la zone d'étude}

D'un point de vue morphologique l'estuaire de Morlaix comprend deux parties (figure 1):

- une section amont longue de $5,5 \mathrm{~km}$, composée de deux bras, l'un menant au déversoir d'un bassin de plaisance situé au cœur de la ville de Morlaix, l'autre constitué par la rivière du Dourduff. Ces estuaires sont peu profonds et marqués par des chenaux étroits bordés de larges vasières découvrantes, de telle sorte que leur largeur moyenne décroit d'un facteur 10 entre Pleine et Basse Mer (de 100 à $10 \mathrm{~m}$ en amont). Les apports fluviaux sont faibles $\left(20 \mathrm{~m}^{3} / \mathrm{s}\right.$ en crue) ;

- l'estuaire extérieur, ou baie, long de $5 \mathrm{~km}$ et large de 3,5 $\mathrm{km}$, en communication avec la Manche par un étroit goulet. L'estran y est très vaste et largement exploité pour la conchyliculture.

L'amplitude de la marée incidente atteint $9,5 \mathrm{~m}$ en vive eau. Compte tenu du caractère fermé de la baie et de la présence de nombreux îlots vers le large, la houle n'y pénètre que très peu : seul un clapot de faible amplitude et de très courte période peut se manifester dans la baie.

\section{Calcul hydrodynamique}

\subsection{Description des modèles}

La marée étant le principal facteur de circulation, un calcul de courant barotrope a été mené en résolvant les équations du mouvement intégrées selon la verticale. Deux modèles ont été réalisés (SAlomon et PommepuY, 1990), l'un bidimensionnel en plan horizontal $(2 \mathrm{DH})$ couvrant la baie et un secteur aval ouvert de façon à calculer les échanges dans le goulet de Penn Al Lann; l'autre est unidimensionnel (IDH) et comprend deux branches : les estuaires du Dourduff et de Morlaix. Le modèle 2DH prend en compte les zones découvrantes par glissement automatique des frontières terrestres au cours des calculs. Le modèle 1DH décrit l'écoulement moyen en chaque section des estuaires et respecte la variabilité effective des sections mouillées et rayons hydrauliques en fonction du niveau de la surface libre. En outre, une distinction est faite entre les sections actives qui participent à l'écoulement et les sections passives qui n'interviennent que dans la conservation de la masse d'eau (stockage de l'eau sur les rives latérales lorsqu'elles sont inondées).

Les systèmes d'équations sont résolus en différences finies. La maille de calcul a une dimension de $250 \mathrm{~m}$ et les conditions aux limites sont les débits fluviaux en amont et la hauteur d'eau le long de la frontière maritime à l'aval. Par ailleurs les modèles $2 \mathrm{DH}$ et $1 \mathrm{DH}$ sont couplés par l'information " cote de la surface libre " grâce au chevauchement de leurs grilles de calcul.

\subsection{Principaux résultats}

En raison des incertitudes sur les conditions à la limite aval, seuls les résultats obtenus au sud de Penn Al Lann sont considérés. La figure 2 qui présente des champs de courant en flot et en jusant met en évidence le rôle prééminent du chenal axial dans les transferts de masse d'eau. On peut noter que les courants convergent vers ce chenal en jusant et divergent en flot : les gradients qui en résultent favorisent la dispersion des éléments dissous. Les vitesses atteignent 1 nœud en flot et 0,9 nœud en jusant, conduisant à des excursions de marée de même échelle que la baie. Sur les banquettes latérales les vitesses sont environ deux fois plus faibles, mais présentent une dissymétrie flot/jusant inverse de celle du chenal. Ainsi ce dernier apparaît à dominante de flot tandis que les bords ont une résiduelle de jusant: ce phénomène bien connu est lié à l'énergie cinétique acquise par la masse d'eau entrant dans le goulet de Penn Al Lann (Salomon et Pommepuy, 1990).

Plus en amont l'assymétrie flot/jusant s'accentue en raison de la déformation de l'onde marée lorsqu'elle se propage par de très faibles profondeurs. Ainsi la figure 3 met en évidence le retard de la marée en amont de l'estuaire au moment de la Basse Mer. En outre on observe dans ce secteur que toute la fin du jusant est marquée par un écoulement fluvial, le niveau de basse mer étant nettement plus bas que la cote du chenal. 

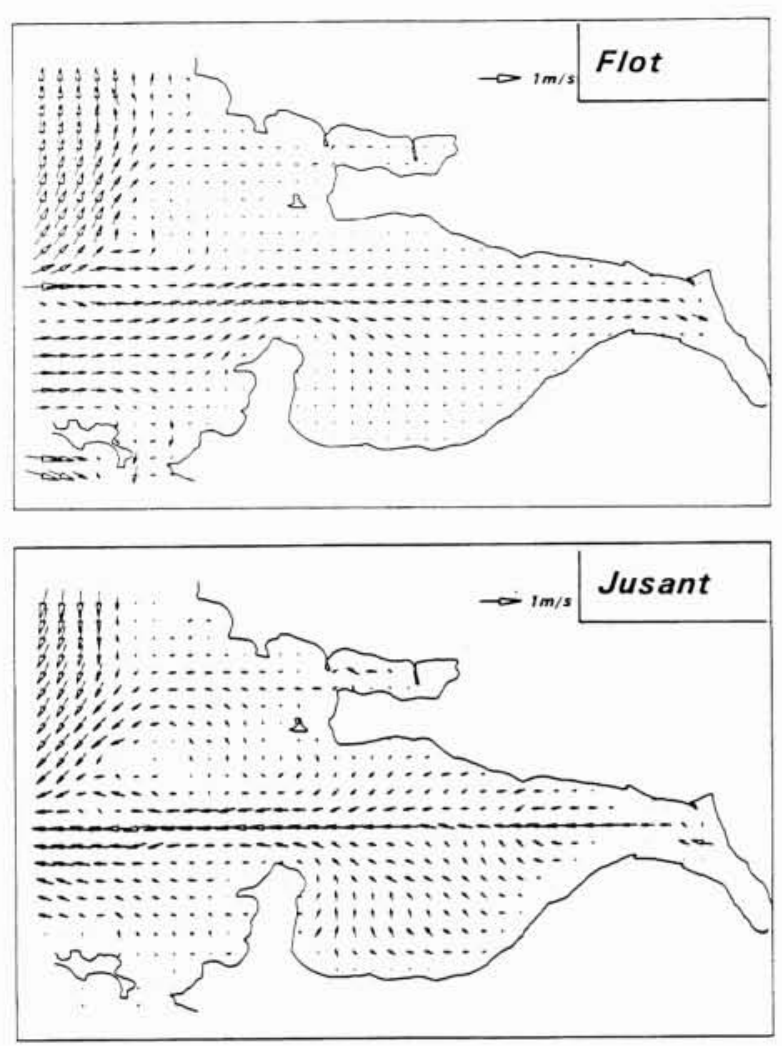

2. Champs de courants calculés dans la baie de Morlaix en vive eau.

3. Propagation de la marée et évolution du courant dans l'estuaire amont de Morlaix.
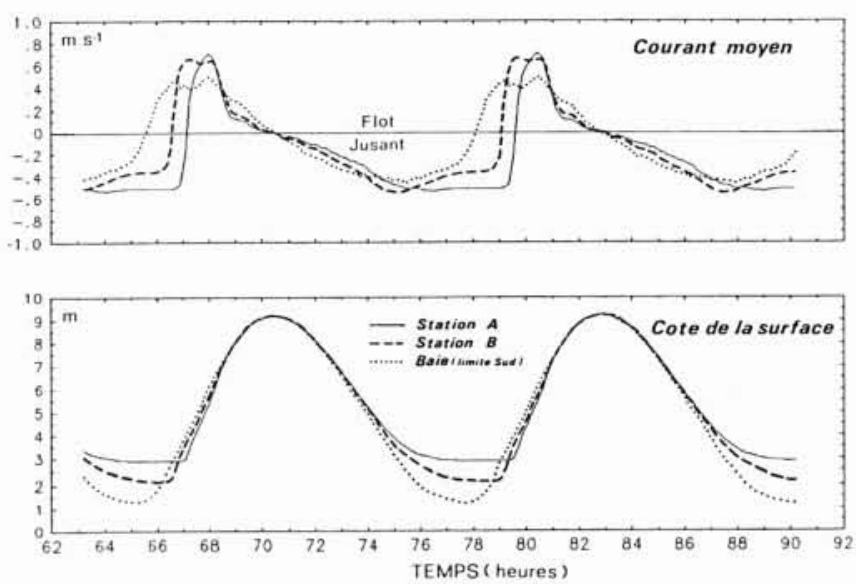

\section{Modèle de transport de sédiment cohésif}

\subsection{Description du modèle}

Le modèle de transport sédimentaire repose sur la conservation de la masse de sédiment, à la fois dans la colonne d'eau sous forme de matière en suspension (M.E.S.) et dans les sédiments superficiels (LE HIR et al., 1989a). Ainsi dans l'eau on résout une équation d'advection/dispersion analogue à l'équation de transport d'un élément dissous, avec en plus des termes de dépôts (D) et d'érosion (E). Cette équation est intégrée selon la verticale et est résolue sur le même maillage que le modèle hydrodynamique $(1 \mathrm{DH}$ ou $2 D H$ ).

Le taux de dépôt est représenté par l'expression de Krone, $D=W s . C f .\left(1-\tau / \tau_{D}\right)$, où $W s$ est la vitesse de chute et Cf la concentration près du fond, déduite de la concentration moyenne à l'aide d'une fonction de forme. Le taux d'érosion est donné par la formule de Partheniades, $E=M .\left(\tau / \tau_{\mathrm{E}}-1\right) \cdot M$ est un coefficient.

$\tau$ est la tension de frottement sur le fond. Elle est déduite du courant moyen en faisant l'hypothèse d'un profil vertical logarithmique des vitesses. Mais dans le modèle $1 \mathrm{DH}$, pour rendre compte de la disparité des échanges avec le sédiment au sein d'une même section transversale, en particulier en présence de larges estrans, des valeurs locales de $\tau$, correspondant à différentes positions du sédiment par rapport à la surface, ont été calculées en supposant une proportionalité entre $\tau$ et la hauteur d'eau locale.

$\tau_{\mathrm{D}}$ et $\tau_{\mathrm{F}}$ sont respectivement les tensions critiques de dépôt et d'érosion. La première est reliée à la concentration en M.E.S. pour tenir compte des phénomènes de floculation tandis que la seconde dépend des propriétés rhéologiques du sédiment superficiel. Selon de nombreux auteurs (Migniot, Owen, Thorn et Parsons, Hayter in Mehta, 1984) on peut écrire $\tau_{\mathrm{E}}=\alpha \mathrm{C}_{\mathrm{sol}}{ }^{\beta}$ où $C$ sol est la concentration du sédiment. Pour cette raison le calcul des M.E.S. est couplé au modèle de sol dont l'algorithme de tassement permet d'évaluer Csol.

$\mathrm{Ce}$ modèle de sol gère un certain nombre de couches dont seule la masse par unité de surface est fixée. Le tassement est pris en compte sous la forme d'une équation différentielle relative à la concentration en sédiment $\mathrm{sec}$; l'intérêt de la méthode est qu'à l'inverse de la plupart des autres modèles de consolidation, elle ne nécessite pas l'ajustement de profils types de concentration (LE HIR et al., 1989b).

La discrétisation du fond sédimentaire est tridimensionnelle, même dans les estuaires oủ l'on a considéré plusieurs banquettes de vases, caractérisée chacune par une cote moyenne, comme le montre la section $M M^{\prime}$ ' de la figure $l$. Pour chacun de ces niveaux de vase, une hauteur d'eau et un frottement local sont estimés et les échanges avec la masse d'eau sont calculés. 

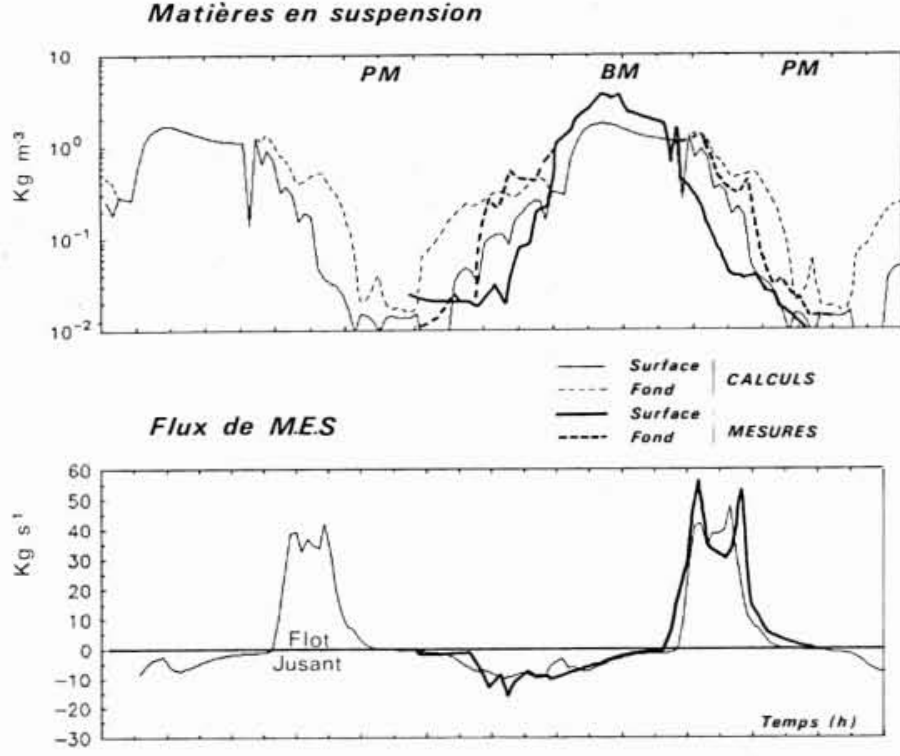

4. Evolution en vive eau des matières en suspension et de leurs flux simulés ou mesurés à la station $\mathrm{B}$.

\subsection{Exemples de résultats}

La figure 4 montre l'évolution des concentrations et flux de M.E.S. au point $B$ pendant 2 marées de vive eau, en période d'étiage. La simulation a commencé 14 jours auparavant de telle sorte qu'un cycle complet Vive Eau/Morte Eau s'est déroulé depuis les conditions initiales. On note un bon accord entre les mesures et les calculs malgré une gamme de variation très large.

Les maxima de concentration obtenus à partir de la Pleine Mer sont successivement l'érosion locale, le passage en fin de jusant des matières érodées en amont, le retour de ce dernier maximum juste après la renverse de flot et enfin l'érosion locale en flot. L'importance du maximum de basse mer est liée aux très faibles sections d'écoulement à ce moment, ainsi qu'à une érosion amont par écroulement des banquettes de vase vers le chenal. Ce phénomène observé en nature (L'YAVANC et BASSOULLET, 1990) a été introduit de manière empirique dans le modèle.

La représentation des flux tempère l'importance relative de ces maxima (en particulier à basse mer où les sections sont très faibles) et laisse apparaitre un flux résiduel de flot. Ainsi l'estuaire amont s'envase pendant l'étiage et il faut attendre un régime de crue pour inverser la tendance (L'YAvanc et BASSOUllet, 1990). Ce flux résiduel de flot se justifie moins par l'assymétrie du courant flot/jusant que par les écarts de durée des renverses de Pleine et Basse Mer (figure 3) et finalement la forte probabilité de dépôt des matériaux à PM sur les banquettes de vase supérieures : celles-ci étant déjà émergées lorsqu'apparaît un fort courant de jusant, les matériaux fraîchement déposés ne peuvent être remis en suspension.

Les applications du modèle de transport en suspension sont nombreuses, en particulier en terme de bilan sédimentaire. Mais ici on s'intéresse au transport de sédiment comme vecteur potentiel de contaminant.

\section{Un logiciel de transport de contaminants dissous et particulaires}

Les différents modèles que nous venons de présenter ont été couplés et intégrés dans un même logiciel de simulation du devenir de plusieurs contaminants, avec interactions éventuelles.

Dans l'eau, le code numérique résout simultanément l'équation de transport de plusieurs "variables d'état". Celles-ci peuvent être des contaminants dissous ou particulaires, parfois présents sous les deux formes; généralement l'une des variables est la concentration totale de M.E.S. qui n'est donc pas considérée à part. Quand il y a interaction entre deux variables, elle est introduite dans les équations de conservation de masse sous forme différentielle: ainsi même lorsque l'adsorption/désorption d'un contaminant suit une loi de partage, une loi du premier ordre avec une cinétique quasi infinie est introduite pour chacune des formes de ce contaminant. Ces interactions sont généralement traitées de manière explicite, ce qui peut induire quelques contraintes sur le pas de temps lors de la résolution numérique.

Dans le sédiment, la consolidation n'est exprimée que pour une seule variable : la concentration du sédiment. Les autres variables (particulaires) sont représentées par leurs masses totales dans chaque couche : ainsi le modèle mémorise la part des contaminants contenue dans le sédiment et rend compte de leurs dépôts ou remise en suspension éventuels (LE Hir et al., 1989c).

Naturellement les processus de dégradation ou croissance de chaque variable peuvent être introduits dans l'eau ou le sédiment.

\section{Application du logiciel au devenir des bactéries}

\subsection{Etude des temps de mortalité des bactéries}

Selon l'heure de la marée, l'effluent à son arrivée en estuaire rejette directement des bactéries, soit dans l'eau où elles se dispersent, soit à basse mer sur le sédiment qui se trouve ainsi directement contaminé.

Afin d'évaluer la mortalité des bactéries fécales dans les deux types d'environnement, des études ont été réalisées sur le sédiment (in vitro) et dans l'eau (in situ) à l'aide d'une même souche de Escherichia coli. Cette souche, représentative des coliformes fécaux, est classiquement utilisée dans les études de mortalité.

Etude de la mortalité dans le sédiment (LE GuYADER, 1989): Les travaux ont été réalisés en laboratoire dans un pilote maintenu à température constante. Les prélèvements de sédiment ont été effectués de manière à en conserver la structure, et la mise en œuvre des expérimentations a eu lieu dans les heures qui suivaient le prélèvement.

Les observations montrent que même en l'absence d'agitation, les échanges entre l'eau et le sédiment sont immédiats. Après deux jours de contact, le système atteint un état d'équilibre avec : 
- une survie importante de la souche dans le sédiment ; - une diminution de la population dans l'eau.

En fin d'expérimentation, les concentrations observées dans le sédiment sont environ 100 fois plus élevées que celles de l'eau. Ainsi les taux de mortalité, caractérisés par les T90 (temps nécessaires pour que $90 \%$ des bactéries meurent), sont très longs dans le sédiment: 14 jours en moyenne avec une valeur maximale de 40 jours.

Etudes de la mortalité dans l'eau (SAlomon et PommePUY, 1990) : Ces études ont été réalisées dans l'estuaire à l'aide de chambres à diffusion immergées à $30 \mathrm{~cm}$ sous la surface. Les expérimentations ont été faites à deux endroits différents, l'un en eau marine, l'autre en eau estuarienne turbide, en été et en hiver.

La figure 5 présente quelques exemples de résultats ainsi obtenus. Les T90 trouvés se répartissent de la façon suivante:

- en aval, en eau de mer, ils varient de 2 heures (avec un bon éclairement en surface) à 2 jours (en surface par temps couvert, ou en profondeur). Les résultats de T90 égaux à quelques heures sont en accord avec ceux précédemment trouvés dans la littérature et qui correspondaient à des travaux réalisés en eau de mer dans des conditions d'éclairement optimales ;

- en amont, dans les eaux turbides, les T90 sont généralement très longs : 6 à 9 jours. Ces valeurs sont dues aux conditions environnementales ; en effet dans les eaux turbides les matières organiques sont riches en éléments favorables à la survie des bactéries fécales (nutrients, osmoprotecteurs). D'autre part la turbidité diminue la pénétration de la lumière qui a un effet bactéricide certain. Ces deux facteurs se surajoutent pour créer des conditions favorables à la survie des bactéries en milieu estuarien.

\subsection{Simulation du rejet bactérien}

Le logiciel de transport multivariable a été appliqué au calcul de la distribution des bactéries fécales issues du rejet de la station d'épuration de Morlaix, situé en amont de l'estuaire. La simulation a été réalisée dans les conditions suivantes :

- rejet à flux constant, conforme aux mesures, avec 50\% de bactéries libres (assimilables à un élément dissous) et $50 \%$ de bactéries liées (au comportement analogue à celui des suspensions);

- simulation de deux cycles complets vive eau/morte eau, pour un débit fluvial moyen $\left(2 \mathrm{~m}^{3} / \mathrm{s}\right)$.

Deux cas ont été considérés : un comportement conservatif des bactéries (sans croissance ni mortalité) et une dégradation par mortalité estimée d'après les résultats des travaux cités en 6.1 à savoir : un T90 de 2 jours pour les bactéries libres, un T90 de 7 jours pour les bactéries liées en suspension et une survie infinie pour les bactéries liées lorsqu'elles sont dans le sédiment.

Les résultats sont présentés au point fixe $\mathrm{B}$ (fig. 6) :

- cas 1 , absence de mortalité : les bactéries libres varient dans une même gamme en vive eau et en morte eau avec

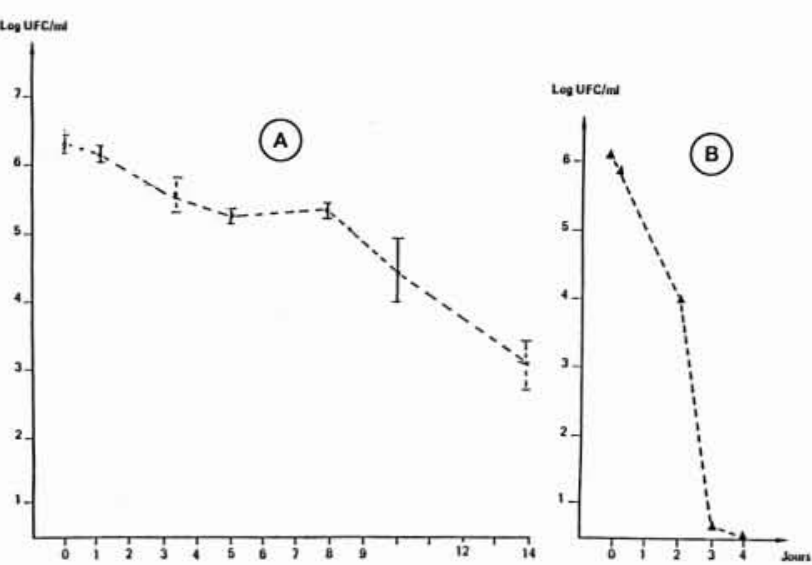

5. Exemples d'évolutions bactériennes (E. coli $H$ 10407) mesurées dans des chambres à diffusion.

a. Mesure en zone turbide (confluent du Dourduff).

b. Mesure au "large" (limite nord de la baie).
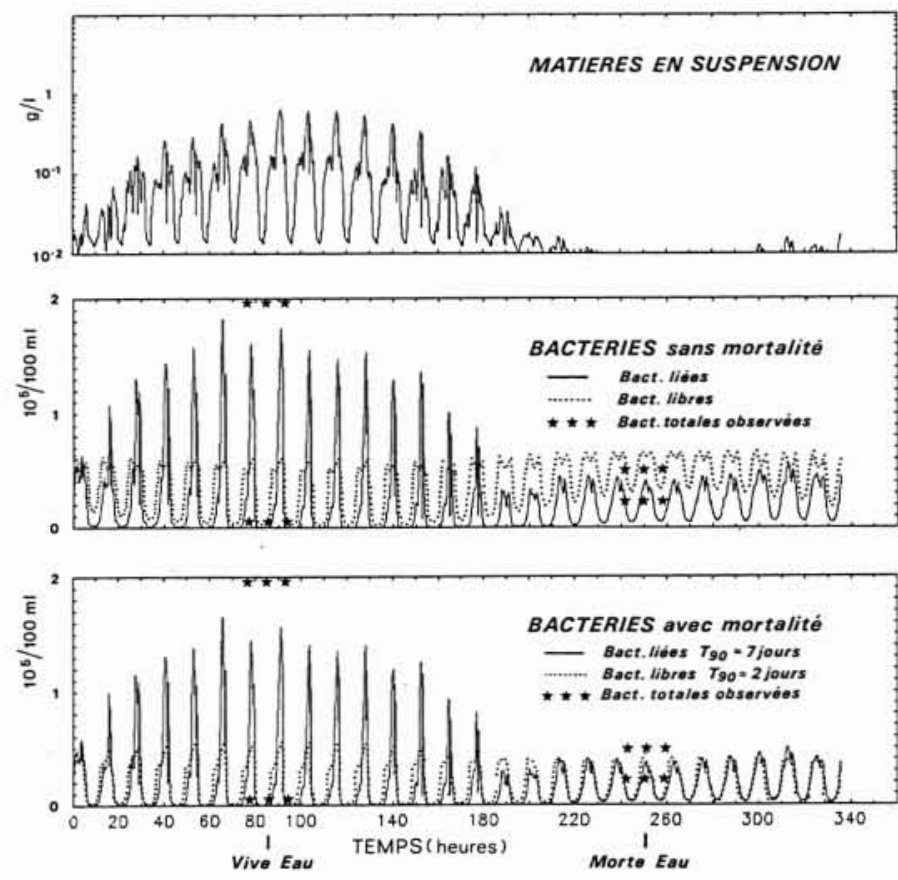

6. Simulation des rejets de bactéries dans l'estuaire de Morlaix. Evolution à la station $B$.

une moyenne supérieure en morte eau compte tenu du temps de séjour plus long en cette période. Au contraire les bactéries liées présentent des variations nettement supérieures en vive eau avec des maxima à basse mer, provenant de l'érosion des matériaux dans un secteur amont proche du rejet ;

- cas 2, prise en compte des mortalités : l'effet est nettement plus sensible sur les bactéries libres pour lesquelles le T90 est relativement court. Ainsi on note qu'en morte eau les bactéries libres et liées seraient en nombre équivalent avec des variations analogues tandis qu'en vive eau l'effet des remises en suspension pendant le jusant reste très sensible.

Ces résultats supportent remarquablement la comparaison avec les observations in situ de bactéries «totales " (les bactéries libres et liées n'étant pas différenciées dans les comptages). Naturellement des processus d'adsorption/désorption sont susceptibles de modifier la réparti- 
A) sans mortalité :
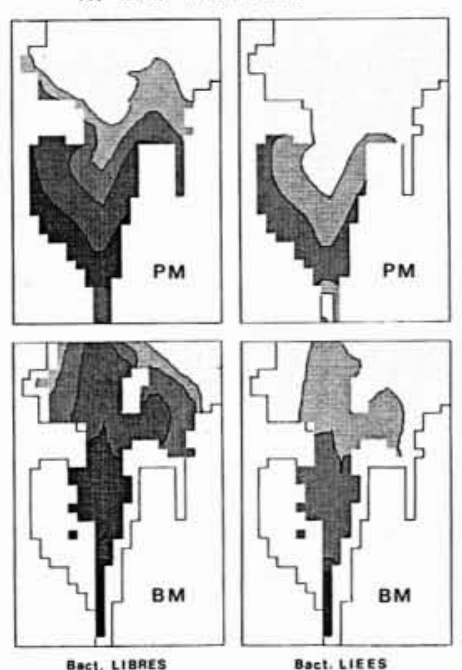

B) avec mortalité :
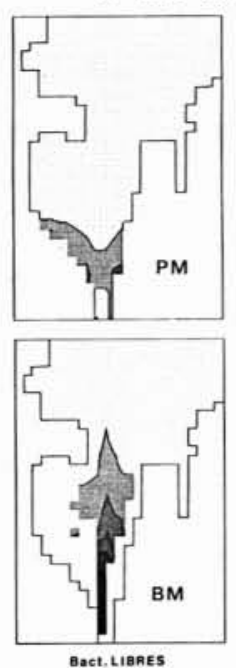

8.CL, LIBRES
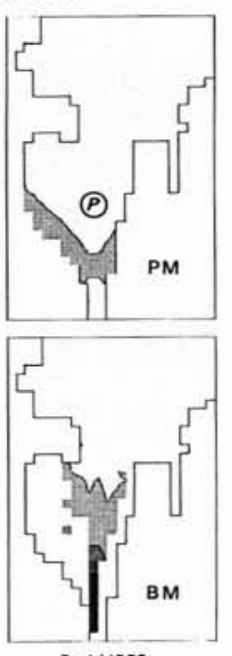

Bact HEFS

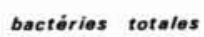

observées en P:

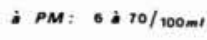

C $B$ M : $102400 / 100 \mathrm{mt}$

7. Simulation des rejets de bactéries dans l'estuaire de Morlaix. Distributions dans la baie au cours d'une marée de vive eau.

tion de ces bactéries libres et liées: faute de mesure les concernant, ils n'ont pas été introduits dans le modèle.

La figure 7 présente les répartitions de bactéries libres et liées plus en aval dans la baie de Morlaix, à pleine mer (PM) et basse mer (BM) d'une marée de vive eau. On peut remarquer qu'en l'absence de mortalité (fig. $7 a$ ) les bactéries libres sont nettement plus nombreuses que les liées, en raison d'un très faible taux de MES. Cependant lorsque les T90 sont introduits (fig. $7 b$ ) les différences sont réduites, compte tenu de la survie accrue des bactéries liées, en particulier lorsqu'elles sont déposées. Les concentrations bactériennes ainsi obtenues par simulation sont du même ordre de grandeur que les mesures, mais la mortalité semble un peu excessive. Il ne s'agit encore que d'une simulation schématique, des améliorations pouvant être attendues grâce à l'introduction d'une mortalité dépendante de la turbidité (donc fonction des MES) et de l'éclairement (reproduction du cycle nycthéméral).

\section{Conclusion}

Le logiciel de transport des contaminants dissous et/ou particulaires que nous avons présenté résulte de l'intégration de plusieurs modèles physiques ou sédimentologiques. Par introduction aisée de lois de comportement non conservatif et d'interactions entre variables, il constitue la base d'un outil de simulation des problèmes de qualité d'eau. En outre chaque composante de ce logiciel peut évoluer au gré de l'état de l'art et en fonction des questions posées. Ainsi le calcul hydrodynamique et la résolution de l'équation de transport pourront à court terme être menés dans les trois dimensions spatiales. De même les processus sédimentaires pourront être affinés, en particulier les lois de tassement des vases. Il faut d'ailleurs rappeler que ces processus sont mal connus et qu'un important travail de réglage du modèle sédimentaire, s'appuyant sur de nombreuses mesures in situ et en laboratoire reste néces saire. Ceci étant, une fois le modèle sédimentaire calibré, il devient opérationnel pour traiter le devenir des contaminants particulaires, comme l'a montré l'application au devenir des bactéries en baie de Morlaix.

Remerciements: Les auteurs tiennent à remercier E. Dupray, P. Bassoullet, J. L'Yavanc, A. Derrien, M. Breton, D. Guillerm et J.P. Annezo pour leurs contributions à ce travail.

\section{Références bibliographiques}

Le GuyAder F., 1989. Colonisation bactérienne et implantation de E. coli dans le sédiment d'origine littorale. Thèse de Doctorat, Université de Rennes I, UER Médicales et Pharmaceutiques.

Le Hir P., Bassoullet P., L'Yavanc J., 1989a. « Modelling mud transport in a macrotidal estuary " in " Advances in Water Modelling and Measurement ", Ed. PALMER, Pub. BHRA, The Fluid Engineering Centre, Bedford, U.K.

Le Hir P., Bassoullet P., L'yavanc J., 1989b. «Newdevelopments about mud transport models : application to a macrotidal estuary ". Compte rendu de l'International Symposium on Sediment Transport Modeling. New Orleans, Aug. 14-18, 1989. Ed. Sam S.Y. WAng, Publication de l'ASCE.

Le Hir P., Guillaud J.F., Salomon J.C., 1989c. " Applications of a cohesive sediment model to dissolved and particulate contaminants transport ". Compte rendu du $23^{c}$ congrès de I'AIRH, Ottawa, 21-25 août 1989.

L'yavanc J., Bassoullet P., 1990. "Nouvelle approche dans l'étude de la dynamique sédimentaire des estuaires macrotidaux à faible débit fluvial». Colloque international "Environnement des mers épicontinentales". Lille, mars 1990. A paraître dans Oceanologica Acta.

Menta A.J., 1984. Editeur de "Estuarine Cohesive Sediment Dynamics ». Springer Verlag. 14, 1984.

SAlomon J.C., Pommepuy M., 1990. « Mathematical model of bacterial contamination of the estuary of Morlaix (France) ". Pour publication dans Water Research. 